\title{
A comprehensive study of long-term skeletal changes after spinal cord injury in adult rats
}

\author{
Tiao Lin ${ }^{1,2 *}$, Wei Tong ${ }^{1,3 *}$, Abhishek Chandra ${ }^{1}$, Shao-Yun Hsu ${ }^{4}$, Haoruo Jia ${ }^{1,5}$, Ji Zhu ${ }^{1}$, Wei-Ju Tseng ${ }^{1}$, Michael A Levine ${ }^{6}$, \\ Yejia Zhang ${ }^{1,7}$, Shi-Gui Yan ${ }^{8}$, X Sherry Liu ${ }^{1}$, Dongming Sun ${ }^{4}$, Wise Young ${ }^{4}$ and Ling Qin ${ }^{1}$
}

Spinal cord injury (SCI)-induced bone loss represents the most severe osteoporosis with no effective treatment. Past animal studies have focused primarily on long bones at the acute stage using adolescent rodents. To mimic chronic SCI in human patients, we performed a comprehensive analysis of long-term structural and mechanical changes in axial and appendicular bones in adult rats after SCI. In this experiment, 4-month-old Fischer 344 male rats received a clinically relevant T13 contusion injury. Sixteen weeks later, sublesional femurs, tibiae, and $\mathrm{L} 4$ vertebrae, supralesional humeri, and blood were collected from these rats and additional non-surgery rats for micro-computed tomography $(\mu \mathrm{CT})$, micro-finite element, histology, and serum biochemical analyses. At trabecular sites, extreme losses of bone structure and mechanical competence were detected in the metaphysis of sublesional long bones after SCI, while the subchondral part of the same bones showed much milder damage. Marked reductions in bone mass and strength were also observed in sublesional $\mathrm{L} 4$ vertebrae but not in supralesional humeri. At cortical sites, SCI induced structural and strength damage in both sub- and supralesional long bones. These changes were accompanied by diminished osteoblast number and activity and increased osteoclast number and activity. Taken together, our study revealed site-specific effects of SCI on bone and demonstrated sustained inhibition of bone formation and elevation of bone resorption at the chronic stage of SCI.

Bone Research (2015) 3, 15028; doi:10.1038/boneres.2015.28; published online: 27 October 2015

\section{INTRODUCTION}

Osteoporosis is a well-known secondary complication of spinal cord injury (SCI). ${ }^{1-2}$ Shortly after the injury, sublesional bone density and mass decline rapidly and linearly. This is particularly deleterious to the cancellous bone located in the metaphyseal-epiphyseal area of the distal femora and proximal tibiae, which experiences a $1 \%-4 \%$ per month bone loss in the first 6-12 months after SCl. ${ }^{3-5}$ This rate is 4-, 10-, and 30-fold greater than those observed during microgravity, prolonged bed rest, and early menopause, respectively. Hence, severe osteoporosis, with at least a $40 \%$ reduction in bone mineral content, is common in $\mathrm{SCl}$ patients. ${ }^{6}$ Studies in patients demonstrate that serum or urine levels of bone resorption markers, such as type I collagen Ctelopeptide and $\mathrm{N}$-telopeptide, increase within 2 weeks post-SCl and reach extremely high levels within 2-4 months. ${ }^{7}$ On the other hand, bone formation markers are at normal or slightly above normal levels during this acute phase..$^{7-8}$ These data suggest that excessive bone resorption is the major contributor for the rapid bone loss in the acute phase.

During the chronic phase that begins 1-2 years after injury, bone mass reaches a nadir at a very low level ${ }^{9-10}$ but bone loss continues. ${ }^{11}$ As a result, $50 \%$ of $\mathrm{SCl}$ patients will sustain a low-impact or osteoporotic fracture at some

'Department of Orthopaedic Surgery, Perelman School of Medicine, University of Pennsylvania, Philadelphia, PA, USA; ${ }^{2}$ Department of Musculoskeletal Oncology, The First Affiliated Hospital of Sun Yat-sen University, Guangzhou, China; ${ }^{3}$ Department of Orthopaedics, Union Hospital, Tongji Medical College, Huazhong University of Science and Technology, Wuhan, Hubei, China; ${ }^{4}$ W.M. Keck Center for Collaborative Neuroscience, Rutgers, The State University of New Jersey, Piscataway, NJ, USA; ${ }^{5}$ Department of Orthopaedic Surgery, School of Medicine, Shihezi University, Shihezi, Xinjiang, China; ' ${ }^{6}$ Division of Endocrinology and Diabetes, The Children's Hospital of Philadelphia, and Department of Pediatrics, Perelman School of Medicine, University of Pennsylvania, PA, USA; ${ }^{7}$ Department of Physical Medicine \& Rehabilitation, Perelman School of Medicine, University of Pennsylvania and Translational Musculoskeletal Research Center, Philadelphia Veterans Affairs Medical Center, Philadelphia, PA, USA and ${ }^{8}$ Department of Orthopaedic Surgery, 2nd Affiliated Hospital, School of Medicine, Zhejiang University, Hangzhou, China

*These authors contributed equally to this work.

Correspondence: L Qin (qinling@mail.med.upenn.edu)

Received: 04 June 2015; Revised: 18 August 2015; Accepted: 20 August 2015 
point following their injury. ${ }^{4}$ Minor stress or trauma, such as transfers, range of motion exercises, bending, or minor falls, can cause a pathological fracture. These sublesional fractures are difficult to treat and are often associated with delayed union and nonunion. ${ }^{12-13}$ In addition to limiting mobility and predisposing to skin pressure ulcers, fractures and their management might trigger severe hypertensive crisis due to autonomic dysreflexia.

To date, there have been many studies using the rat $\mathrm{SCl}$ model to understand the mechanisms of SCl-induced bone damage and to explore possible treatments. However, there are several limitations associated with the previous studies that employed the $\mathrm{SCl}$ model, which reduce their potential clinical relevance. First, most of the studies used adolescent rats ranging from 4 to 8 weeks of age. Morse et al. have demonstrated that $\mathrm{SCl}$ also damages the growth plates of long bones in young rats (7 weeks old) by disorganizing the chondrocyte columnar structure, decreasing chondrocyte number, and accelerating chondrocyte apoptosis. ${ }^{14}$ This growth plate arrest is well correlated with clinical cases of children with $\mathrm{SCl}$ who have stunted bone growth below the injury site. ${ }^{15}$ However, the average age of human subjects at the time of $\mathrm{SCl}$ is 43 years, when growth plates have been closed for a long time and bone mass has passed its peak amount. Therefore, it should be more appropriate to perform experiments on adult rats to minimize the confounding factor of longitudinal bone growth. Secondly, most previous studies focused on analyzing bone changes at the acute stage. Because $\mathrm{SCl}$ is a lifelong condition, it would be most useful to delineate bone structural and cellular changes during the chronic stage of $\mathrm{SCl}$ and to investigate potential therapies that might improve bone strength at this stage. Lastly, there have been few and conflicting animal data characterizing the $\mathrm{SCl}$ damage on supralesional bones, particularly at the chronic stage. Besides disuse and bone denervation, other systemic factors, such as hormonal modifications, may also play pivotal roles in the development of osteoporosis after SCl. ${ }^{2}$ To date, the clinical outcome of $\mathrm{SCl}$ on forearm bone mass remains uncertain. ${ }^{16-18}$

To prevent rapid bone loss, hypercalciuria, and sometimes hypercalcemia after $\mathrm{SCl}$, antiresorptive drugs, such as bisphosphonates, are frequently administered to reduce high bone resorption. While these drugs decrease bone loss in SCl patients, they have not been shown to increase BMD in any skeletal sites tested. ${ }^{3,19-20}$ In addition, long-term use of bisphosphonates is associated with atypical femur fractures, which might worsen the condition of $\mathrm{SCl}$ patients. Mechanical loading, such as weight-bearing and functional electrical stimulation (FES), is promising if applied during the early stage of $\mathrm{SCl}$ but its effect at the later stage is unknown, with minor or no bone benefits reported. ${ }^{21-22}$
To delineate the damaging effect of $\mathrm{SCl}$ on the entire skeleton in a clinically relevant setting, we performed a comprehensive analysis of long-term structural and mechanical changes in axial and appendicular bones in adult male rats after $\mathrm{SCl}$. Our study revealed site-specific effects of $\mathrm{SCl}$ on bone and demonstrated sustained inhibition of bone formation and elevation of bone resorption at the chronic stage of $\mathrm{SCl}$. These results provide mechanistic insight for developing new effective treatments for $\mathrm{SCl}$ induced severe osteoporosis.

\section{METHODS}

$\mathrm{SCl}$ surgery and tissue harvest

All animal studies were reviewed and approved by the Institutional Animal Care and Use Committees (IACUC) at Rutgers University and the University of Pennsylvania. Fourmonth-old male Fischer 344 rats (Taconic, Hudson, NY, USA, $n=5$ ) were anesthetized by isoflurane and their back skin was incised at the mid-line. Muscle was sharply dissected from the spinal column to expose the T9-10 dorsal processes. After cutting the dorsal intravertebral ligament, a T9-10 laminectomy was used to expose the T13 cord. Rats were then moved to a New York University Impactor where their T13 spinal cord received modest injury delivered by a 10-g rod dropped from a $25 \mathrm{~mm}$ height. Muscle and skin were then closed with stitches and stainless steel clips, respectively. Post-surgery, manual bladder expression was performed 1-2 times per day throughout the entire experiment. The control group consisted of age- and gendermatched Fischer 344 rats $(n=6)$. Sixteen weeks later, rats were weighed, subjected to cardiac puncture for blood collection, and perfused with $4 \%$ paraformaldehyde (PFA). Sublesional bones, including femurs and tibiae, and supralesional bones, including fourth lumbar vertebrae (L4) and humeri, were collected and fixed further in 4\% PFA for subsequent measurements. Before fixation, the full lengths of long bones were measured by a Vernier caliper.

Evaluation of bone microarchitecture by microcomputed tomography

All bones were scanned by a compact fan-beam-type vivaCT40 (Scanco Medical AG, Bassersdorf, Switzerland) at a $15 \mu \mathrm{m}$ nominal voxel size. The scanned and analyzed areas of each type of bone are summarized in Table 1. All images were first smoothed by a Gaussian filter (sigma = 1.2 , support $=2.0$ ) and then thresholded corresponding to 392.1 (trabecular bone) and $582 \mathrm{mgHA} \cdot \mathrm{cm}^{-3}$ (cortical bone). Three-dimensional analyses were performed to calculate trabecular parameters, such as trabecular volumetric bone mineral density (VBMD), bone volume fraction $\left(\mathrm{BV} \cdot \mathrm{TV}^{-1}\right.$ ), trabecular thickness (Tb.Th), trabecular number (Tb.N), trabecular separation (Tb.Sp), and structure model index (SMI), and cortical parameters, such as cortical 
Table 1. $\mu \mathrm{CT}$-analyzed areas for measuring trabecular and cortical structural parameters in appendicular and axial bones at 16 weeks after SCI

\begin{tabular}{lll}
\hline Bone & \multicolumn{1}{c}{ Scanned area } & Analyzed area \\
\hline Femur & Subchondondral bone & $2-3 \mathrm{~mm}$ below the distal growth plate within the secondary ossification center \\
Femur & Primary spongiosa & $1-2.5 \mathrm{~mm}$ above the distal growth plate within the primary ossification center \\
Femur & Secondary spongiosa & $2.5-4 \mathrm{~mm}$ above the distal growth plate within the primary ossification center \\
Femur & Mid-shaft cortical bone & $0.5 \mathrm{~mm}$ above to $0.5 \mathrm{~mm}$ below the midline of a femur \\
Humerus & Secondary spongiosa & $0.75-2.25 \mathrm{~mm}$ below the proximal growth plate within the primary ossification center \\
Humerus & Mid-shaft cortical bone & $4.5-5.25 \mathrm{~mm}$ below the proximal growth plate within the primary ossification center \\
L4 vertebra & Trabecular bone & $1.5 \mathrm{~mm}$ below the top growth plate to $1.5 \mathrm{~mm}$ above the bottom growth plate \\
\hline
\end{tabular}

BMD, cortical area (Ct.Ar), cortical thickness (Ct.Th), periosteal perimeter (Ps.Pm), endocortical perimeter (Ec.Pm), porosity, and polar moment of inertia (pMOl) as described by Bouxsein et al. ${ }^{23}$

Trabecular bone stiffness was calculated for humeri, femora, and L4 vertebrae based on thresholded microcomputed tomography $(\mu \mathrm{CT})$ images as previously described. ${ }^{24}$ Briefly, microstructural finite element ( $\left.\mu \mathrm{FE}\right)$ models were generated by converting each bone voxel to an 8-node brick element. Bone tissue was modeled as an isotropic, linear elastic material with a Young's modulus of 15 GPa and a Poisson's ratio of 0.3. A uniaxial compression was applied along the axial direction of the model and the model was subjected to a linear elastic analysis to determine the bone stiffness.

\section{Bone histology analysis}

After $\mu \mathrm{CT}$ scans, right tibiae were decalcified and processed for paraffin sections. Five- $\mu \mathrm{m}$ longitudinal sections were stained either by hematoxylin and eosin (H\&E) for counting the number of cuboidal and plump bone lining osteoblasts, or by tartrate-resistant acid phosphatase (TRAP) assay kit (Sigma-Aldrich, St. Lovis, MO, USA) for counting the number of TRAP-positive multinucleated osteoclasts within the secondary spongiosa. All images were captured by Nikon Eclipse 90i and quantified using Bioquant Osteo Software (Bioquant Image Analysis, Nashville, TN, USA).

\section{Serum chemistry}

Blood was collected via cardiac puncture at euthanasia and left at room temperature for at least 30 min before centrifuging at $200 \times \mathrm{g}$ for $10 \mathrm{~min}$ to separate serum. Serum calcium levels were measured by Calcium Colorimetric Assay (Sigma-Aldrich). Osteocalcin and TRACP 5b level were determined by Rat Osteocalcin EIA Kit (Biomedical Technologies, Stoughton, MA, USA) and RatTRAP $^{T M}$ Assay (Immunodiagnostic Systems, Scottsdale, $A Z$, USA), respectively.

\section{Statistics}

Data are expressed as means \pm standard error (SEM) and analyzed by unpaired, two-tailed Student's t-test for comparison between control and SCl groups using Prism 5 soft- ware (GraphPad Software, San Diego, CA, USA). Values of $P<0.05$ were considered statistically significant.

\section{RESULTS}

\section{General observations}

Initial body weight was the same among control (308 \pm 15 g) and SCl (305 $\pm 14 \mathrm{~g}$ ) groups. Sixteen weeks after surgery, the weight of SCl rats $(289 \pm 15 \mathrm{~g})$ was $30 \%(P<0.001)$ lower than that of controls $(417 \pm 8 \mathrm{~g})$. Both sublesional and supralesional long bone lengths were significantly affected in SCl rats compared to controls (femur: control $41.5 \pm$ $0.2 \mathrm{~mm}, \mathrm{SCl} 37.8 \pm 0.9 \mathrm{~mm}, P<0.01$; humerus: control $31.6 \pm 0.2 \mathrm{~mm}, \mathrm{SCl} 29.1 \pm 0.5 \mathrm{~mm}, P<0.01$ ). These results suggest that $\mathrm{SCl}$ impairs normal weight gain and longitudinal bone growth.

Bone microarchitectural impairment in sublesional extremities after $\mathrm{SCl}$

In SCl patients, the most severe bone loss occurs at the knee joint, including both distal femur and proximal tibia. There are three types of trabecular bone within this region: primary and secondary spongiosa in the metaphyseal area and subchondral trabecular bone in the epiphyseal area. All three types of bone are formed through endochondral ossification. During development, the two metaphyseal sites are formed within the primary ossification center and the epiphyseal site is formed within the secondary ossification center. While most previous studies focused on analyses of trabecular bone in the secondary spongiosa, there are very few reports describing the changes in trabecular bone at the other two sites under physiological and pathological conditions. To gain detailed knowledge about SCl damage on bone in the knee joint, all three sites were scanned by $\mu \mathrm{CT}$ at a high resolution at 16 weeks post-surgery. At the distal femoral site, we observed that the most drastic bone loss occurred in the secondary spongiosa immediately followed by the primary spongiosa, while, surprisingly, there was only modest bone loss in the subchondral region (Figure 1a). Specifically, 3D analysis of $\mu C T$ data revealed striking $54 \%$ and $65 \%$ reductions in $\mathrm{VBMD}$ and $\mathrm{BV} \cdot \mathrm{TV}^{-1}$, respectively, in the secondary spongiosa from the SCI group compared to those from controls (Figure 1b). This was mainly due to significant decreases in Tb.N (36\%) and Tb.Th (26\%), and an 
a

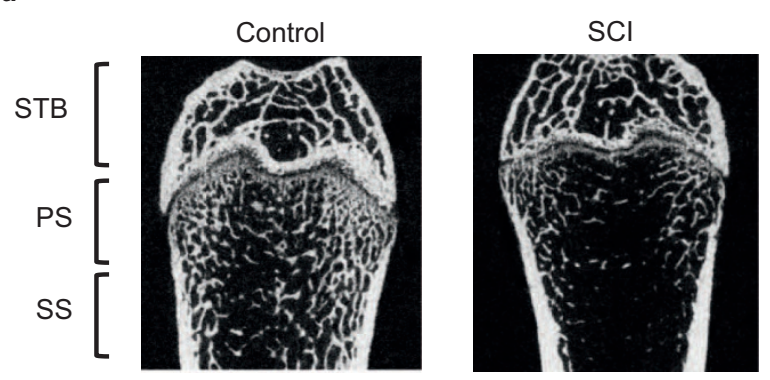

b
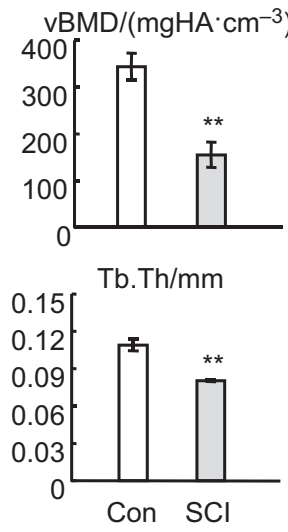

d

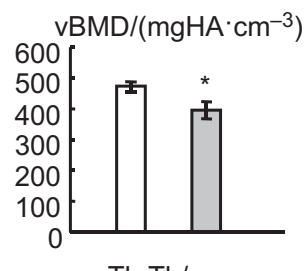

$\mathrm{Tb} . \mathrm{Th} / \mathrm{mm}$

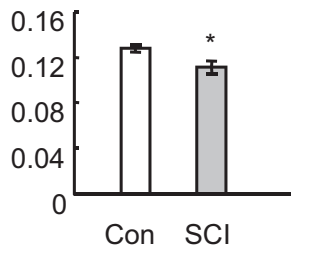

Con
$\mathrm{BV} \cdot \mathrm{TV}^{-1}$

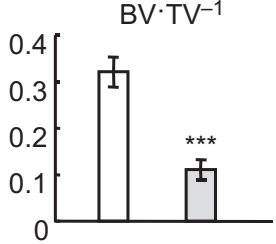

$\mathrm{Tb} . \mathrm{Sp} / \mathrm{mm}$

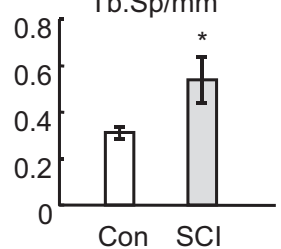

$\mathrm{BV} \cdot \mathrm{TV}^{-1}$

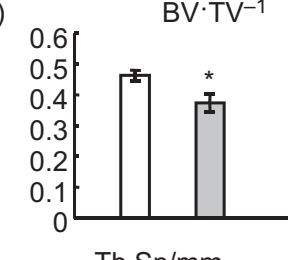

Tb.Sp/mm

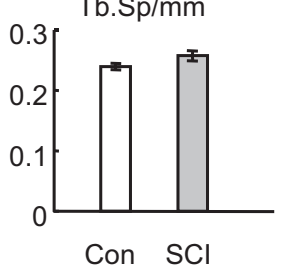

Tb. N/mm -1

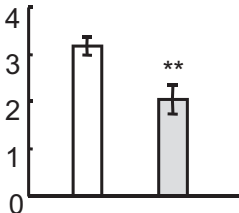

SMI

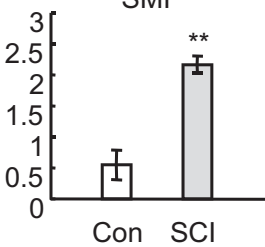

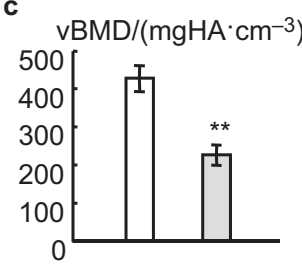

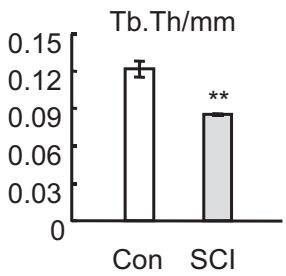

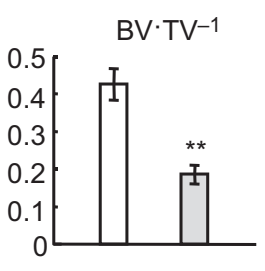

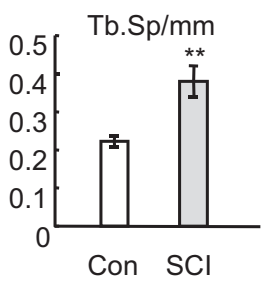

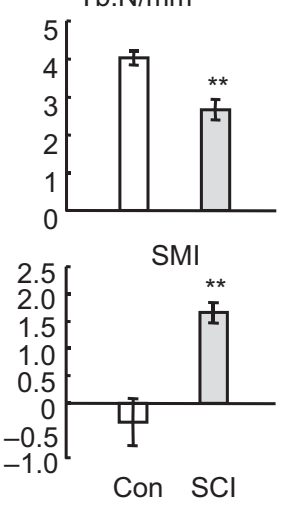
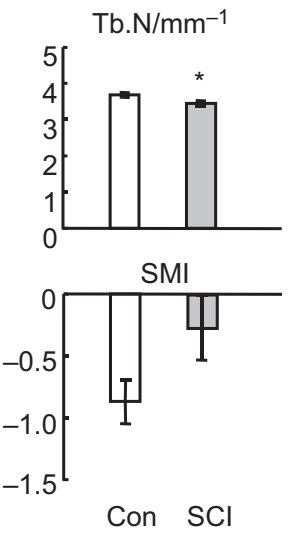

Figure 1. SCI causes severe trabecular bone loss and structural deterioration in the metaphyseal area but relatively moderate damage in the subchondral bone area in distal femur. (a) Representative longitudinal $\mu$ CT images of distal femurs in control and SCI rats at 16 weeks after injury. Brackets define the regions of three types of trabecular bone: subchondral trabecular bone (STB), primary spongiosa (PS), and secondary spongiosa (SS) in the metaphysis. (b) $\mu \mathrm{CT}$ measurement of trabecular structural parameters in the secondary spongiosa area. (c) $\mu \mathrm{CT}$ measurement of trabecular structural parameters in the primary spongiosa area. (d) $\mu \mathrm{CT}$ measurement of trabecular structural parameters in the subchondral trabecular area. ${ }^{*} \mathrm{P}<0.05$; $* * P$ $<0.01 ;{ }^{* * *} P<0.001$ vs control (con).

increase in Tb.Sp (73\%). Furthermore, a 2.9-fold increase in SMI suggests that $\mathrm{SCl}$ impairs the structural integrity of trabecular bone. In the primary spongiosa, similar but relatively smaller reductions in $\mathrm{VBMD}(47 \%), \mathrm{BV} \cdot \mathrm{TV}^{-1}(56 \%)$, Tb.N (34\%), Tb.Th (30\%), and Tb.Sp (70\%), and an increase in SMI were observed in SCl rats compared to controls (Figure 1c). By contrast, much milder trabecular bone damage $\left(-16 \%\right.$ in $\mathrm{VBMD},-19 \%$ in BV. $\mathrm{TV}^{-1},-6 \%$ in Tb.N, $-13 \%$ in $\mathrm{Tb} . \mathrm{Th},+7 \%$ in Th.Sp, and $+69 \%$ in SMI) was detected in the subchondral area (Figure 1d), implying that the extent of trabecular bone loss induced by $\mathrm{SCl}$ is site-specific even within the same bone. Similar patterns of trabecular bone damage were also observed in the proximal tibial region (data not shown).

Next, we analyzed cortical bone structural parameters at the femoral mid-shaft. As shown in Figure 2, cortical BMD remained the same after SCl. However, compared to those from the control group, bones from the SCl group were slimmer with $14 \%$ and $8 \%$ reductions in Ps.Pm and Ec.Pm, respectively, resulting in a $16 \%$ decrease in Ct.Th and a $30 \%$ decrease in Ct.Ar. These structural changes led to a drastic $45 \%$ reduction in $\mathrm{pMOl}$, a parameter reflecting the bending strength of cortical bone.

The effects of $\mathrm{SCl}$ on bone microarchitecture in forelimbs In addition to the paralysis of trunk and lower extremities below the injury site, $\mathrm{SCl}$ causes remarkable neuronal and hormonal changes throughout the entire body, which might affect the bones in the upper extremities. To explore this in our SCl rat model, the supralesional proximal humeri were scanned and analyzed by $\mu \mathrm{CT}$. We did not detect any differences in trabecular bone within the subchondral site and the primary spongiosa after $\mathrm{SCl}$ (Figure 3a). In the secondary spongiosa, a trend of structural impairment was observed, with $15 \%, 20 \%, 21 \%$, and $4 \%$ decreases in $\mathrm{VBMD}$, BV.TV ${ }^{-1}, \mathrm{~Tb} . \mathrm{N}$, and Tb.Th, respectively, and a $29 \%$ increase in Tb.Sp (Figure $3 b$ ) in the SCl group compared to controls, but none of the changes reached statistical significance. By contrast, we observed significant changes in the mid-shaft cortical bone of humeri in SCl rats (Figure $3 \mathrm{c}$ and $3 \mathrm{~d}$ ). Bone perimeters, Ps.Pm and Ec.Pm, decreased by $9 \%$ and $12 \%$, respectively, in the $\mathrm{SCl}$ group compared to controls, but 
a
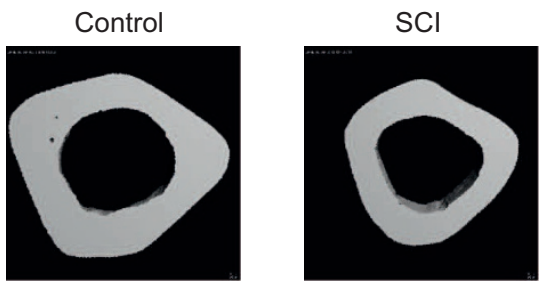

b
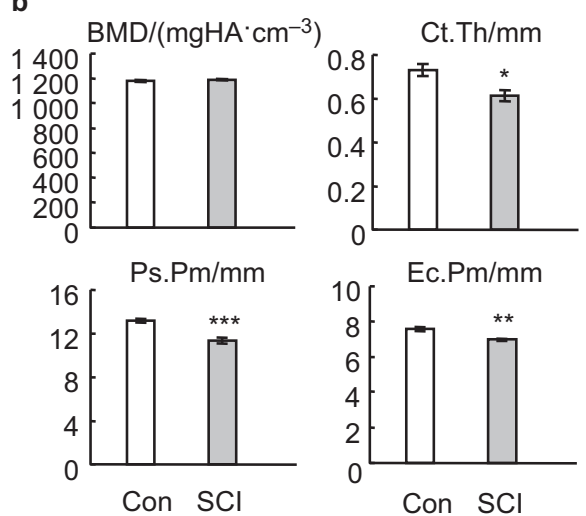

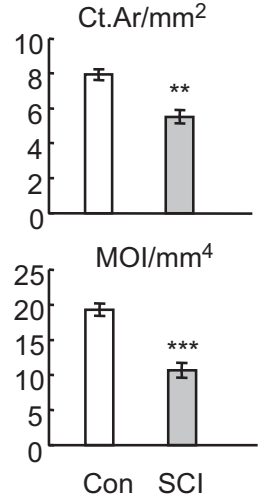

Figure 2. SCI alters cortical bone structure and impairs cortical bone strength in femur. (a) Representative cross-sectional $\mu \mathrm{CT}$ images of femoral mid-shaft in control and SCI rats at 16 weeks after injury. (b) $\mu \mathrm{CT}$ measurement of cortical structural parameters and bending strength. ${ }^{*} P<0.05 ;{ }^{* *} P<0.01 ;{ }^{* * *} P<0.001$ vs con. bone thickness (Ct.Th) increased by $9 \%$. A small but significant increase in cortical BMD (3\%) was also detected after $\mathrm{SCl}$. Overall, those changes led to a trend of decrease in bending strength after $\mathrm{SCl}$ (a 17\% decrease in pMOI, $P=$ 0.056). Taken together, our results indicate that $\mathrm{SCl}$ also has harmful effects on the mobilized forelimbs, albeit to a much lesser extent compared to affected immobilized hindlimbs.

\section{$\mathrm{SCl}$ is detrimental to vertebral trabecular bone}

Clinical studies describe conflicting conclusions about whether sublesional axial bones are as severely affected by SCl as appendicular bones. ${ }^{25-26}$ In our study, SCl rats lost significant amounts of trabecular bone in the sublesional L4 vertebral body, particularly within the central region (Figure $4 \mathrm{a}$ ). Trabecular $\mathrm{VBMD}$ and $\mathrm{BV} \cdot \mathrm{TV}^{-1}$ in $\mathrm{L} 4$ from SCl rats were $31 \%$ and $37 \%$, respectively, less than those from controls, and were accompanied by remarkable decreases in Tb.N (16\%) and Tb.Th (27\%) as well as increases in Tb.Sp (32\%) and SMI (Figure 4b).

The site-specific effect of $\mathrm{SCl}$ on trabecular bone strength $\mu \mathrm{FE}$ analysis was performed to measure the integrated mechanical competence of trabecular compartments in femur, humerus, and vertebra (Figure 5). Strikingly, the a

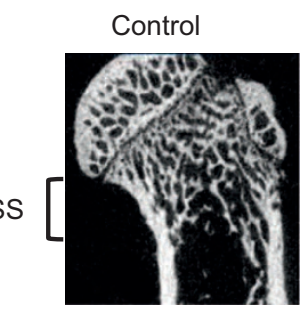

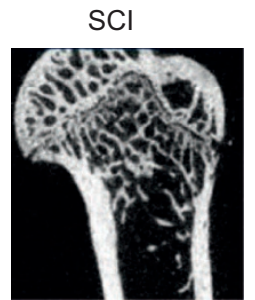
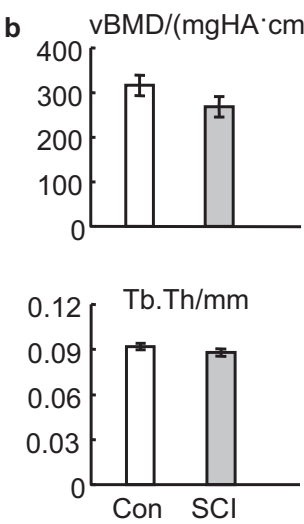

$\mathrm{BV} \cdot \mathrm{TV}^{-1}$
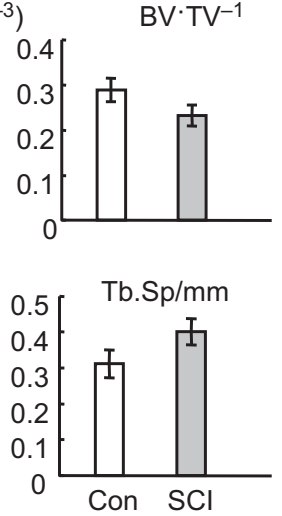

Tb. $\mathrm{N} / \mathrm{mm}^{-1}$
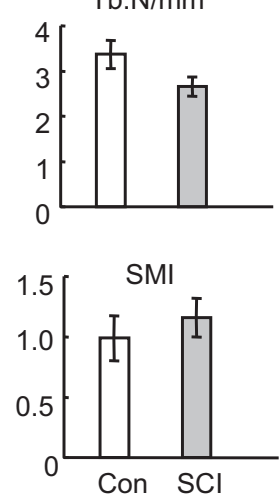

c
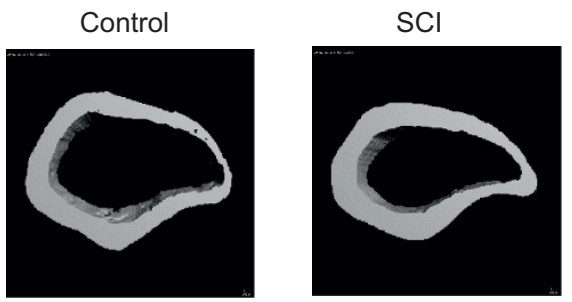

d
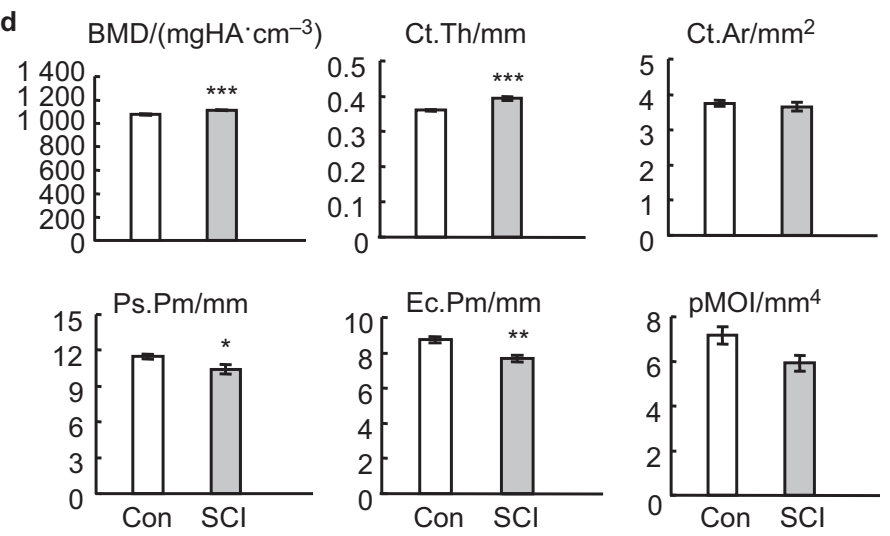

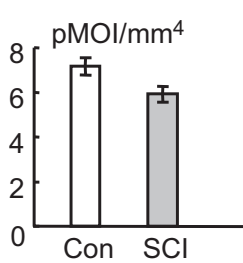

Figure 3. SCI results in a trend of trabecular bone loss and significant cortical bone damage in supralesional humerus. (a) Representative longitudinal $\mu \mathrm{CT}$ images of proximal humeri in control and SCI rats at 16 weeks after injury. Brackets define the regions of the secondary spongiosa (SS). (b) $\mu \mathrm{CT}$ measurement of trabecular structural parameters in the secondary spongiosa area. (c) Representative cross-sectional $\mu \mathrm{CT}$ images of humeral mid-shaft in control and SCI rats at 16 weeks after injury. (d) $\mu \mathrm{CT}$ measurement of cortical structural parameters and bending strength. ${ }^{*} P<0.05$; ${ }^{* *} P<0.01$; $* * * P<0.001$ vs con. 
a
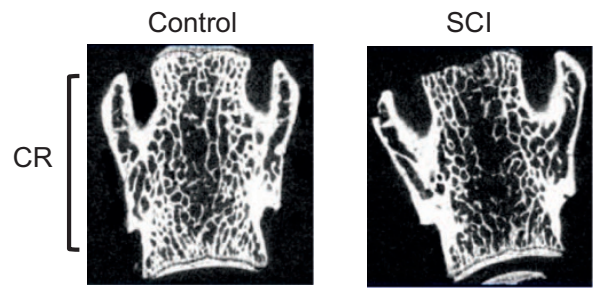

b
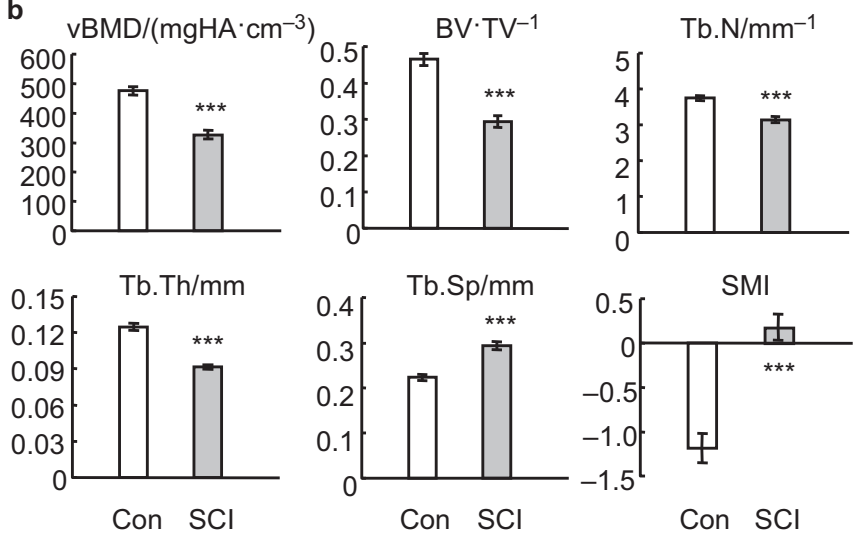

Figure 4. The vertebral trabecular bone is impaired by SCI. (a) Representative longitudinal $\mu \mathrm{CT}$ images of L4 vertebrae in control and SCI rats at 16 weeks after injury. Brackets define the central trabecular region of vertebra (CR). (b) $\mu \mathrm{CT}$ measurement of trabecular structural parameters inside the vertebra. ${ }^{* * *} P<0.001$ vs con.

loss of trabecular bone and deterioration of bone structure led to an almost complete diminishment of bone stiffness $(91 \%)$ in the femoral secondary spongiosa from SCl rats compared to controls. In line with the above results that $\mathrm{SCl}$ causes a trend of trabecular bone impairment in humerus, we detected only a non-significant decrease in trabecular bone stiffness in this region after SCl. However, strong reduction in bone stiffness (52\%) was observed in L4 vertebra.

\section{Bone histology and serum biochemistry analysis}

To understand the underlying cellular mechanisms of longterm SCl damage to bone, we performed histological analyses on right tibiae. At 16 weeks post-surgery, osteoblast number was $71 \%$ less and osteoclast number was 3.7-fold more than those in controls (Figure 6a). This imbalance

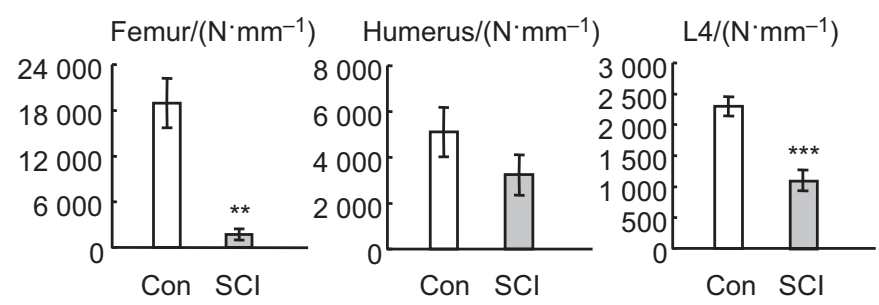

Figure 5. SCI has site-specific effects on trabecular bone stiffness. Based on a $\mu \mathrm{FE}$ model, bone stiffness was calculated from the secondary spongiosa area of femur and humerus and from the L4 vertebral trabecular bone. ${ }^{* *} P<0.01 ;{ }^{* * *} P<0.001$ vs. con. a

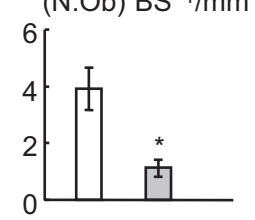

b

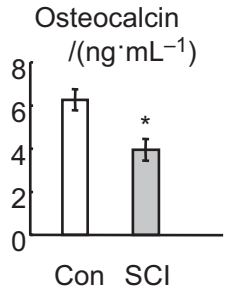

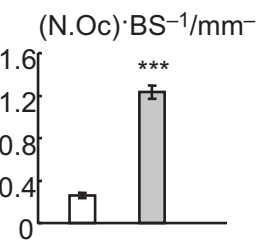

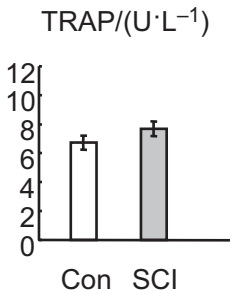

Serum calcium

16 $/\left(\mathrm{mg} \cdot \mathrm{dL}^{-1}\right)$

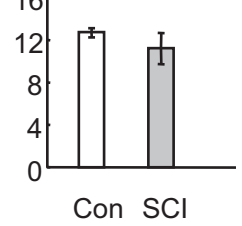

Figure 6. Chronic SCI inhibits bone formation and stimulates bone resorption. (a) Histological analysis was performed to count osteoblast number and osteoclast number per bone surface in tibial metaphyseal area from control and SCI rats at 16 weeks after injury. (b) Biochemical assays of osteocalcin, TRAP, and serum calcium level in control and SCI groups. ${ }^{*} P<0.05 ;{ }^{* * *} P<0.001$ vs con.

between bone-forming and bone-resorbing cells was further attested by a decreased serum level of bone-formation marker (osteocalcin) and a trend of increased amount of resorption marker (TRAP; Figure 6b). Serum calcium level is usually elevated at the acute $\mathrm{SCl}$ stage due to the hyperactivation of osteoclasts (14). However, it returned to normal in SCl rats at this chronic stage (Figure 6b).

\section{DISCUSSION}

Bone is a dynamic tissue that undergoes constant remodeling, and coordination between osteoblastic and osteoclastic activities is required for optimal bone homeostasis. After $\mathrm{SCl}$, the interaction between osteoblasts and osteoclasts favors bone resorption, leading to severe bone loss particularly in the sublesional appendicular bones and increased risk of low impact fractures in these bones. In this study, we used an adult rat T13 contusion model to reproduce the effects of $\mathrm{SCl}$ in mature patients and performed a comprehensive analysis of the long-term effects of $\mathrm{SCl}$ on bone structure and mechanics at multiple clinically relevant skeletal sites. Our high resolution $\mu \mathrm{CT}$ scans revealed that the most severe trabecular bone loss and structural deterioration occurred in the metaphyseal area of tibia and femur. By contrast, more modest but significant damage occurred in vertebral trabecular bone, and only a trend of trabecular bone loss was noted in humerus. Further $\mu \mathrm{FE}$ analysis confirmed this sequence in terms of the degree of mechanical property changes. At cortical sites, $\mathrm{SCl}$ does not induce bone demineralization but reduces bone amount and strength in both sub- and supralesional bones. Many factors, including local ones (immobilization and bone denervation) and systemic ones (hormonal and metabolic changes), contribute to 
SCl-induced bone damage. Our data indicate that trabecular bone is primarily sensitive to local factors while cortical bone is also sensitive to systemic factors.

Remarkably, we found that $\mathrm{SCl}$ has distinct site-specific effects on trabecular bone even within the same appendicular bone. In mammals, long bone is developed through endochondral ossification and eventually has two ossification centers. ${ }^{27}$ The primary ossification center is formed in the center of diaphysis at the embryonic stage by converting the hypertrophic cartilage in the growth plate into trabecular bone and marrow space. It consists of primary spongiosa, which is directly connected with the growth plate and is mainly shaped by bone modeling during longitudinal growth, and secondary spongiosa, which is derived from the primary spongiosa by a bone remodeling process. Shortly after birth, the epiphyseal cartilage is excavated by canals invaginated from the perichondrium to form the secondary ossification center. The trabecular bone within this center is anatomically located below the articular cartilage and is termed subchondral bone. Previous studies have principally examined pathological effects of $\mathrm{SCl}$ on trabecular bone in the metaphyseal region, whereas no studies have attempted to characterize the response of subchondral bone. Surprisingly, we found that subchondral bone is much more resistant to $\mathrm{SCl}$ damage than trabecular bone in the metaphyseal area. Moreover, unlike the other trabecular sites, where the bone loss is caused by both trabecular thinning and loss of trabecular bone, the SClinduced subchondral bone loss occurs mainly through decreasing Tb.Th with little loss of Tb.N. To our knowledge, this is the first report delineating selective responses of trabecular bone within different anatomical regions of the same bone to the same injury. Further investigation will be required to identify whether this is specific for $\mathrm{SCl}$ only or represents a more general response to skeletal perturbations and to define the functional mechanisms.

Because of complicated post-surgery care, the majority of previous rodent studies examined bone phenotypes shortly (i.e., usually within 3 weeks) after SCl. ${ }^{14,28-32}$ The longest follow-up of bone phenotypes was 6 months after $\mathrm{SCl}$ in young (6-week-old) male Sprague-Dawley rats by Jiang et al. ${ }^{33}$ Similarly to our results, they reported a striking $76 \%$ reduction in $\mathrm{BV} \cdot \mathrm{TV}^{-1}$ in tibial metaphysis and a relatively milder $34 \%$ reduction in $L 4$ vertebra at this chronic stage. However, their densitometric analysis and mechanical testing did not detect any changes in distal radius and humerus, respectively, leading to their conclusion that $\mathrm{SCl}$ has a negligible effect on supralesional bones. The discrepancy between our data and theirs on supralesional appendicular bone can be attributed to differences in rat strains (F344 vs Sprague-Dawley), type of SCl model (contusion vs transection), outcome measurement (areal $B M D$ vs volumetric BMD), and animal age (4-month-old vs 6-week-old). While we found that adult SCl rats barely gained weight or even lost weight after paralysis, adolescent $\mathrm{SCl}$ rats almost doubled their body weight after 6 months. ${ }^{33}$ These data imply that $\mathrm{SCl}$ might have greater systemic effects on adult rats than on young rats.

Currently, there is no conventional treatment for SClinduced osteoporosis at the chronic phase. Given the extreme degree of bone loss following $\mathrm{SCl}$, which far exceeds that induced by other insults, such as estrogen deficiency, ${ }^{34}$ neurological deficit, ${ }^{35}$ and mechanical unloading, ${ }^{36}$ and the notable loss of osteoblasts at later stages, antiresorptive agents such as bisphosphonates would seem to be ineffective choices for treating bone loss at chronic SCl. The great reduction (-71\%) in osteoblast number in tibial metaphysis revealed in our study suggests that, in contrast to acute $\mathrm{SCl}$ when the osteoblast number and activity are unaffected or even elevated, ${ }^{37}$ chronic SCl almost completely eliminates the osteoblasts in the metaphyseal region of tibia and femur. Therefore, in order to restore bone mass, to repair micro-architectural damage, and to reduce risk of fracture, anabolic treatments that greatly stimulate new bone formation via promoting osteoblast number and activity should be suitable therapies for $\mathrm{SCl}$ patients at the chronic stage. The treatment choices include teriparatide (recombinant human PTH134), the only FDA-approved anabolic treatment for severe postmenopausal osteoporosis, ${ }^{38}$ and monoclonal antibody against Sclerostin (SCl-Ab), which has shown potent efficacy in patients with postmenopausal osteoporosis in a phase 2 clinical trial. ${ }^{39}$ A recent patient study suggested a trend of bone improvement after 1 year of teriparatide injection together with gait training. However, it did not reach statistical significance, likely due to the limited number of subjects. ${ }^{40}$ In contrast, the preclinical data with Scl$\mathrm{Ab}$ are very promising. Three weeks of Scl-Ab injections immediately after $\mathrm{SCl}$ completely restored the trabecular bone structure and cortical bone strength in rats. ${ }^{41}$

Our study has several limitations. First, we used age- and gender-matched naive rats instead of sham-operated rats as controls. According to our experience, sham operation does not have detectable effects on rat behavior and neuron functions and should not significantly affect our conclusions. Second, unlike human subjects, rats never cease longitudinal bone growth. As shown in our recently published paper, ${ }^{42}$ at age of 8 months, rat proximal tibial growth rate is still at $3 \mu \mathrm{m} /$ day. Therefore, we cannot completely exclude the confounding factor of growth on the metaphyseal region, especially the primary spongiosa. Indeed, we still observed reduced bone length in both femur and humerus. Applying a 3D image registration approach we developed recently ${ }^{24}$ on in vivo longitudinally scanned $\mu \mathrm{CT}$ images should further improve the accuracy and significance of our data. Nevertheless, 
in terms of analyzing bone phenotypes and studying the underlying mechanism, this adult rat model is much more clinically relevant than young adolescent rats.

In conclusion, our comprehensive study has demonstrated that chronic $\mathrm{SCl}$ has deleterious effects on the entire skeleton, with the most severe bone loss and structural deterioration in the lower extremities followed by sublesional vertebrae. The upper extremities also experience bone damage but to a much lesser extent. Furthermore, our finding of sustained inhibition of bone formation and continuous elevation of bone resorption at the chronic stage of $\mathrm{SCl}$ strongly suggest that anabolic treatments should be vigorously pursued for promoting bone health and preventing fractures in patients with chronic SCl.

\section{Acknowledgements}

This work was supported by the National Institutes of Health (R01DK095803 to LQ, 1K08HD049598 to YZ), Penn Center for Musculoskeletal Disorders P30AR050950 (NIAMS/NIH), ASBMR Junior Faculty Osteoporosis Basic Research Award (to LQ), and NIH/NIAMS R03-AR065145 (to XSL). We thank Mr. Sean O'Leary for his invaluable assistance and technical help in all the procedures and animal care. We are grateful to the donors of the Spinal Cord Injury Project for their generous support of spinal cord injury studies at the W. M. Keck Center for Collaborative Neuroscience at Rutgers University. The authors thank Dr. Martin Heyworth for critically editing the manuscript.

\section{References}

1 Battaglino RA, Lazzari AA, Garshick E, Morse LR. Spinal cord injuryinduced osteoporosis: pathogenesis and emerging therapies. Curr Osteoporos Rep 2012; 10: 278-285.

2 Jiang SD, Dai LY, Jiang LS. Osteoporosis after spinal cord injury. Osteoporos Int 2006; 17: 180-192.

3 Gilchrist NL, Frampton CM, Acland RH et al. Alendronate prevents bone loss in patients with acute spinal cord injury: a randomized, double-blind, placebo-controlled study. J Clin Endocrinol Metab 2007; 92: 1385-1390.

4 Szollar SM, Martin EM, Sartoris DJ, Parthemore JG, Deftos LJ. Bone mineral density and indexes of bone metabolism in spinal cord injury. Am J Phys Med Rehabil 1998; 77: 28-35.

5 Warden SJ, Bennell KL, Matthews B, Brown DJ, McMeeken JM, Wark JD. Quantitative ultrasound assessment of acute bone loss following spinal cord injury: a longitudinal pilot study. Osteoporos Int 2002; 13: 586-592.

6 Dauty M, Perrouin Verbe B, Maugars Y, Dubois C, Mathe JF. Supralesional and sublesional bone mineral density in spinal cord-injured patients. Bone 2000; 27: 305-309.

7 Maïmoun L, Couret I, Micallef JP et al. Use of bone biochemical markers with dual-energy $\mathrm{x}$-ray absorptiometry for early determination of bone loss in persons with spinal cord injury. Metabolism 2002; 51: 958-963.

8 Uebelhart D, Hartmann D, Vuagnat H, Castanier M, Hachen HJ, Chantraine A. Early modifications of biochemical markers of bone metabolism in spinal cord injury patients. A preliminary study. Scand J Rehabil Med 1994; 26: 197-202.

9 Eser P, Schiessl H, Willnecker J. Bone loss and steady state after spinal cord injury: a cross-sectional study using pQCT. J Musculoskelet Neuronal Interact 2004; 4: 197-198.

10 Frotzler A, Berger M, Knecht H, Eser P. Bone steady-state is established at reduced bone strength after spinal cord injury: a longitudinal study using peripheral quantitative computed tomography (pQCT). Bone 2008; 43: 549-555.
11 de Bruin ED, Vanwanseele B, Dambacher MA, Dietz V, Stüssi E. Longterm changes in the tibia and radius bone mineral density following spinal cord injury. Spinal Cord 2005; 43: 96-101.

12 Garland DE. Clinical observations on fractures and heterotopic ossification in the spinal cord and traumatic brain injured populations. Clin Orthop Relat Res 1988: 86-101.

13 Nottage WM. A review of long-bone fractures in patients with spinal cord injuries. Clin Orthop Relat Res 1981: 65-70.

14 Morse L, Teng YD, Pham L et al. Spinal cord injury causes rapid osteoclastic resorption and growth plate abnormalities in growing rats (SCI-induced bone loss in growing rats). Osteoporos Int 2008; 19: 645-652.

15 Duval-Beaupere G, Lougovoy J, Trocellier L, Lacert P. Trunk and leg growth in children with paraplegia caused by spinal cord injury. Paraplegia 1983; 21: 339-350.

16 Sabo D, Blaich S, Wenz W, Hohmann M, Loew M, Gerner HJ. Osteoporosis in patients with paralysis after spinal cord injury. A cross sectional study in 46 male patients with dual-energy X-ray absorptiometry. Arch Orthop Trauma Surg 2001; 121: 75-78.

17 Vlychou M, Papadaki PJ, Zavras GM et al. Paraplegia-related alterations of bone density in forearm and hip in Greek patients after spinal cord injury. Disabil Rehabil 2003; 25: 324-330.

18 Zehnder Y, Lüthi M, Michel D et al. Long-term changes in bone metabolism, bone mineral density, quantitative ultrasound parameters, and fracture incidence after spinal cord injury: a cross-sectional observational study in 100 paraplegic men. Osteoporos Int 2004; 15: 180-189.

19 Moran de Brito CM, Battistella LR, Saito ET, Sakamoto H. Effect of alendronate on bone mineral density in spinal cord injury patients: a pilot study. Spinal Cord 2005; 43: 341-348.

20 Zehnder Y, Risi S, Michel D et al. Prevention of bone loss in paraplegics over 2 years with alendronate. J Bone Miner Res 2004; 19: 1067-1074.

21 de Bruin ED, Frey-Rindova P, Herzog RE, Dietz V, Dambacher MA, Stüssi E. Changes of tibia bone properties after spinal cord injury: effects of early intervention. Arch Phys Med Rehabil 1999; 80: 214-220.

22 Zamarioli A, Battaglino RA, Morse LR et al. Standing frame and electrical stimulation therapies partially preserve bone strength in a rodent model of acute spinal cord injury. Am J Phys Med Rehabil 2013; 92: 402-410.

23 Bouxsein ML, Boyd SK, Christiansen BA, Guldberg RE, Jepsen KJ, Müller R. Guidelines for assessment of bone microstructure in rodents using micro-computed tomography. J Bone Miner Res 2010; 25: 1468-1486.

24 Lan S, Luo S, Huh BK et al. 3D image registration is critical to ensure accurate detection of longitudinal changes in trabecular bone density, microstructure, and stiffness measurements in rat tibiae by in vivo microcomputed tomography $(\mu \mathrm{CT})$. Bone 2013; 56: 83-90.

25 Bauman WA, Kirshblum S, Cirnigliaro C, Forrest GF, Spungen AM. Underestimation of bone loss of the spine with posterior-anterior dualenergy X-ray absorptiometry in patients with spinal cord injury. J Spinal Cord Med 2010; 33: 214-220.

26 Bauman WA, Schwartz E, Song IS et al. Dual-energy X-ray absorptiometry overestimates bone mineral density of the lumbar spine in persons with spinal cord injury. Spinal Cord 2009; 47: 628-633.

27 Mackie EJ, Tatarczuch L, Mirams M. The skeleton: a multi-functional complex organ: the growth plate chondrocyte and endochondral ossification. J Endocrinol 2011; 211: 109-121.

28 Yarrow JF, Conover CF, Beggs LA et al. Testosterone dose-dependently prevents bone and muscle loss in rodents following spinal cord injury. $J$ Neurotrauma 2014; 31: 834-845.

29 Wang HD, Shi YM, Li L, Guo JD, Zhang YP, Hou SX. Treatment with resveratrol attenuates sublesional bone loss in spinal cord-injured rats. Br J Pharmacol 2013; 170: 796-806. 
30 Yan J, Li B, Chen JW, Jiang SD, Jiang LS. Spinal cord injury causes bone loss through peroxisome proliferator-activated receptor-gamma and Wnt signalling. J Cell Mol Med 2012; 16: 2968-2977.

31 Medalha CC, Amorim BO, Ferreira JM et al. Comparison of the effects of electrical field stimulation and low-level laser therapy on bone loss in spinal cord-injured rats. Photomed Laser Surg 2010; 28: 669-674.

32 Jiang SD, Jiang LS, Dai LY. Spinal cord injury causes more damage to bone mass, bone structure, biomechanical properties and bone metabolism than sciatic neurectomy in young rats. Osteoporos Int 2006; 17: 1552-1561.

33 Jiang SD, Jiang LS, Dai LY. Changes in bone mass, bone structure, bone biomechanical properties, and bone metabolism after spinal cord injury: a 6-month longitudinal study in growing rats. Calcif Tissue Int 2007; 80: 167-175.

34 Slade JM, Bickel CS, Modlesky CM, Majumdar S, Dudley GA. Trabecular bone is more deteriorated in spinal cord injured versus estrogen-free postmenopausal women. Osteoporos Int 2005; 16: 263-272.

35 Lloyd ME, Spector TD, Howard R. Osteoporosis in neurological disorders. J Neurol Neurosurg Psychiatry 2000; 68: 543-547.

36 Cervinka T, Sievänen H, Hyttinen J, Rittweger J. Bone loss patterns in cortical, subcortical, and trabecular compartments during simulated microgravity. J Appl Physiol 2014; 117: 80-88.
37 Jiang SD, Jiang LS, Dai LY. Effects of spinal cord injury on osteoblastogenesis, osteoclastogenesis and gene expression profiling in osteoblasts in young rats. Osteoporos Int 2007; 18: 339-349.

38 Augustine M, Horwitz MJ. Parathyroid hormone and parathyroid hormone-related protein analogs as therapies for osteoporosis. Curr Osteoporos Rep 2013; 11: 400-406.

39 McClung MR, Grauer A, Boonen S et al. Romosozumab in postmenopausal women with low bone mineral density. N Engl J Med 2014; 370: 412-420.

40 Gordon KE, Wald MJ, Schnitzer TJ. Effect of parathyroid hormone combined with gait training on bone density and bone architecture in people with chronic spinal cord injury. PM R 2013; 5: 663-671.

41 Beggs LA, Ye F, Ghosh P et al. Sclerostin inhibition prevents spinal cord injury induced cancellous bone loss. J Bone Miner Res 2015; 30: 681-689.

42 Altman AR, Tseng WJ, de Bakker CM et al. Quantification of skeletal growth, modeling, and remodeling by in vivo micro computed tomography. Bone 2015: 81: 370-379.

cc) (1) () $\Theta$ This work is licensed under a Creative Commons Attribution(BY No No NonCommercial-NoDerivs 4.0 Unported License. The images or other third party material in this article are included in the article's Creative Commons license, unless indicated otherwise in the credit line; if the material is not included under the Creative Commons license, users will need to obtain permission from the license holder to reproduce the material. To view a copy of this license, visit http://creativecommons.org/licenses/by-nc-nd/4.0/ 